\title{
高价碘试剂的有机电化学合成及应用研究进展
}

\author{
张怀远* 唐蓉萍石星丽颉林伍家卫 \\ (兰州石化职业技术学院应用化学工程学院 甘肃省石油化工过程工程应用技术协同创新中心 兰州 730060)
}

\begin{abstract}
摘要 芳基碘化物的阳极氧化是一种绿色高效的用于合成高价碘试剂的方法, 该方法用电流代替了化学试剂, 避免了 使用价格昂贵、反应后处理棘手的氧化剂, 如 $m$-CPBA, $\mathrm{H}_{2} \mathrm{O}_{2}$, Oxone, Selectfluor 等. 利用电化学合成的高价碘试剂, 既 可促进氟化、氧化环化等反应, 也可成功地应用于天然产物的全合成. 一价碘/三价碘氧化还原体系可用来高效地形成 碳碳键、碳杂原子等化学键. 此外, 能回收循环再利用的芳基碘介质可用于间接阳极氟化, 且容易和产物分离. 总结近 年来高价碘试剂的电化学合成方法及其介导的各种化学反应.
\end{abstract}

关键词 电化学合成; 高价碘试剂; 氟化; 氧化环化

\section{Recent Advances in Organic Electrochemical Synthesis and Application of Hypervalent lodine Reagents}

\author{
Zhang, Huaiyuan* Tang, Rongping Shi, Xingli Xie, Lin Wu, Jiawei \\ (Gansu Province Collaborative Innovation Center of Petrochemical Engineering Application Technology, \\ College of Applied Chemical Engineering, Lanzhou Petrochemical Polytechnic, Lanzhou 730060)
}

\begin{abstract}
Anodic oxidation of aryl iodine compouds is a green and efficient method for the synthesis of hypervalent iodine reagents. This method replaces chemical reagents with electric current, avoiding the use of expensive and handle difficult oxidants such as $m$-CPBA, $\mathrm{H}_{2} \mathrm{O}_{2}$, oxone, selectfluor etc. Electrochemically generated hypervalent iodine reagents can not only promote fluorination, oxidative cyclization, but also be successfully applied in the total synthesis of natural products. In addition, recyclable aryl iodine mediator can be used to indirect anodic fluorination and easily separated from products. The organic electrochemical synthesis of hypervalent iodine reagents and their applications in various chemical transformations are reviewed.

Keywords electrochemical synthesis; hypervalent iodine reagent; fluorination; oxidative cyclization
\end{abstract}

高价态的碘化物被称为高价碘试剂，其分子结构中 没有普通的 $\pi$ 键，而是一个线性的通过碘原子的 $5 \mathrm{p}$ 轨道 与两个配体的 $\mathrm{L}$ 轨道重叠而形成的三中心四电子的化 学键 $(\mathrm{L}-\mathrm{I}-\mathrm{L})$, 称之为 “高价键”。由于 “高价键” 是 一种比较弱并且高度极化的化学键, 所以高价碘试剂具 有特殊的结构特征以及反应活性，在有机合成中应用广 泛 $^{[1]}$, 如烯烃和环丙烷的双官能化 ${ }^{[2]}$ 、酚类化合物的脱芳 构化 ${ }^{[3]}$ 、氧化偶联 ${ }^{[4]}$ 、氧化环化 ${ }^{[5]}$ 、氧化胺基化 ${ }^{[6]}$ 、卤化 [7]、芳基化 ${ }^{[8]}$ 和重排 ${ }^{[9]}$ 等; 利用手性高价碘试剂或手性芳 基碘化物和氧化剂组成的催化体系, 可高对映选择性地

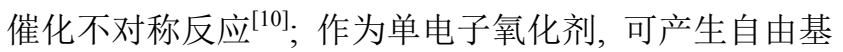

中间体 ${ }^{[11]}$. 高价碘试剂具有反性能多样、容易制备、反 应条件温和、环境友好等优点, 通常代替重金属氧化剂 进行反应. 然而, 其合成需要使用过量的氧化剂 ${ }^{[12]}$, 如 $m$-CPBA, $\mathrm{H}_{2} \mathrm{O}_{2}$, Oxone, Selectfluor 等, 氧化剂不但价格 昂贵，而且反应后处理棘手. 因此，通过单价碘化物的 阳极氧化进行高价碘试剂的电化学合成受到科学家的 广泛关注，这是由于有机电化学合成方法用电流代替了 氧化剂和还原剂，与传统方法相比具有可持续性高、反 应条件温和及化学选择性高等优点 ${ }^{[13]}$. 在过去的 30 年 当中, 出版了很多关于高价碘试剂的书籍和综述 ${ }^{[1 \mathrm{a} \sim \mathrm{hh}}$, 也见证了高价碘试剂的蓬勃发展, 然而高价碘试剂的电

\footnotetext{
* Corresponding author. E-mail: zhanghuaiyuan@lzpcc.edu.cn

Received February 11, 2019; revised March 10, 2019; published online April 9, 2019.

Project supported by the Scientific Research Projects of Colleges and Universities in Gansu Province (No. 2018B-091) and the Teaching and Scientific Research Project of Lanzhou Petrochemical Poly Technic (No. JY2018-25).

甘肃省高等学校科学研究(No. 2018B-091)和兰州石化职业技术学院教科研(No. JY2018-25)资助项目.
} 
化学合成应用综述尚未报道. 本文从二芳基碘盐的电化 学合成、含氟高价碘试剂的电化学合成及其介导的氟化 反应和双(三氟乙氧基)碘苯的电化学合成及其介导的氧 化环化三个方面总结了近年来高价碘试剂的电化学合 成方法及其促进的各种化学反应.

\section{1 二芳基碘盐的电化学合成}

二芳基碘盐是一类重要的高价碘试剂，其分子结构 中有两个碳配体和一个抗衡离子, 通常对空气和水稳 定. 二芳基碘盐既是一类高效的氧化剂, 也是一类高效 的亲电芳基化试剂, 能与含氧、硫、氮、碳的亲核试剂 发生偶联反应，还可作为芳炔前体分子参与有机反 应 $^{[14]}$. 其经典的合成方法通常需要两步反应, 首先, 在 酸的催化下将芳基碘化物氧化生成三价碘试剂, 随后与 芳烃发生偶联反应，对称和不对称的二芳基碘盐均可通 过这一方法来制备 ${ }^{[15]}$. 近年来, 不对称二芳基碘盐的一 锅法和流动化学法的合成也有报道 ${ }^{[16]} .1966$ 年, Miller 和 Hoffmann ${ }^{[17]}$ 报道了在芳烃存在下芳基碘的电化学阳 极氧化, 一步合成了二芳基碘盐. 作者研究了碘苯(1a) 的恒电位氧化, 将 $1 \mathrm{a}$ 溶解于 $0.5 \mathrm{~mol} / \mathrm{L}$ 高氯酸锂的乙腈 溶液中, $1.6 \mathrm{~V}$ ( vs. $\mathrm{Ag} / \mathrm{Ag}^{+}$) 的条件下进行电解, 最终以 47\%的产率得到 4-碘苯苯基高氯酸碘(3a), 利用该方法 分别以 $32 \%$ 和 $56 \%$ 的产率合成了二芳基碘盐 $3 \mathrm{~b}$ 和 $3 \mathrm{c}$ (Eq. 1).

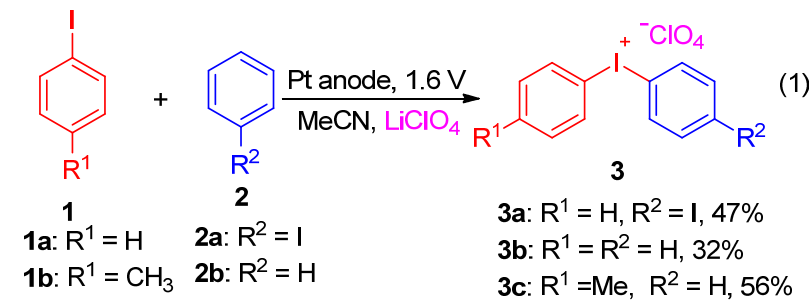

由于苯在 $1.9 \mathrm{~V}$ 以下不能被氧化, 因此, 二芳基碘 盐 3 的形成是来源于芳基碘 $\mathbf{1}$ 的氧化及随后与芳烃 $\mathbf{2}$ 所 发生的偶联. 1975 年, Wendt 小组 ${ }^{[18]}$ 对这一反应进行了 深入地研究, 并提出更合理的反应机理, 他们借助极普 法和动力学研究了苯存在下碘苯在金电极表面上的阳 极氧化, 结果表明反应生成 4-碘苯苯基醋酸碘(5a) 和二 苯基醋酸碘 $(\mathbf{5 b})$ 的产率几乎相等, 即在金电极上发生的 偶联反应没有选择性. 然而, 酸催化下的偶联反应, 选 择性很高(Eq. 2), 生成碘盐 $\mathbf{5 a}$ 和 $\mathbf{5 b}$ 的相对反应速率为 $1: 30$. 两种截然相反的实验结果表明, 电化学生成二 芳基碘盐的机理不是经过三价碘中间体的生成及随后 与芳烃发生偶联的过程, 而是通过在电极上生成自由基 阳离子的中间体, 进而与苯发生偶联, 机理如 Scheme 1 所示.
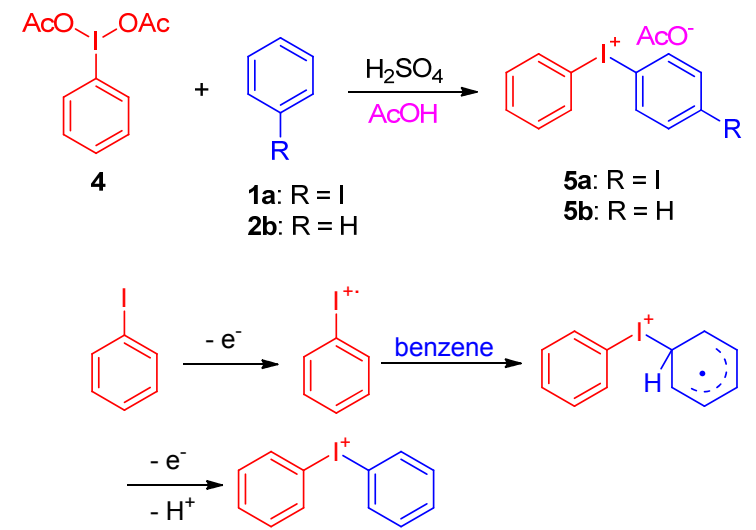

图式 1 碘苯和苯阳极氧化偶联生成二芳基碘盐的机理 Scheme 1 Mechanism of the anodic oxidation of iodobenzene and arene coupling

Pletcher 小组 ${ }^{[19]}$ 证明了上述方法的可行性, 并且利 用烷基取代的芳基碘和芳烃的阳极氧化/偶联，合成了 系列对称和不对称的二芳基碘盐. 方法是以乙酸酎-硫 酸为介质，在以碳毡为阳极、石墨棒为阴极构成的无隔 膜电解槽中进行电解，便以 $48 \%$ ～92\%的产率得到各种 取代的二芳基碘盐化合物. 然而，该方法的局限性在于 底物芳基碘和芳烃不能含有易被氧化的官能团. 另外， 当芳基碘化物含有吸电子基团时，增加了其氧化电位, 不利于阳极氧化; 当芳烃中连有吸电子基团时，降低了 芳烃的亲核性，不利于偶合反应的进行，因此，将 4-硝 基碘苯与甲苯或 4-碘甲苯与硝基苯进行反应，均不能得 到二芳基碘盐. 当芳基碘和芳烃中连有供电子基团时, 既可以降低芳基碘化合物的氧化电位，也可提高芳烃的 亲核性，将有利于反应的顺利进行. 例如，将 4-甲氧基 碘苯(1c) 和苯甲醚 $(2 \mathrm{c})$ 在质量分数为 $2 \%$ 乙酸酐和 $5 \%$ 硫 酸的乙酸电解质中电解(Eq. 3), 以 $42 \%$ 的产率得到 4,4 '二甲氧基二苯基碘盐 6 .

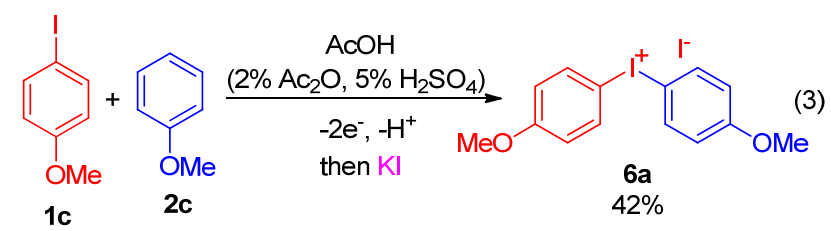

Wirth 等 ${ }^{[20]}$ 利用电化学微反应器成功地开发了一种 用于合成二芳基碘盐的流动化学方法. 微反应器由两个 铂电极构成，电极被一个含有 $250 \mu \mathrm{m}$ 厚的聚全氟异丙 烯箔片隔开. 将溶有芳基碘和芳烃底物的乙腈/醋酸酐/ 硫酸的混合溶液, 用注射洜 $(80 \mu \mathrm{L} / \mathrm{min})$ 通过反应器的进 料口注入，在恒定电流 $30 \mathrm{~mA}$ 下电解，反应结束后，将 反应混合物收集到碘化钾的溶液中，产物二芳基碘盐便 会沉淀析出，通过该方法，以 $18 \% \sim 72 \%$ 的产率合成了 系列二芳基碘盐 8 (Scheme 2). 


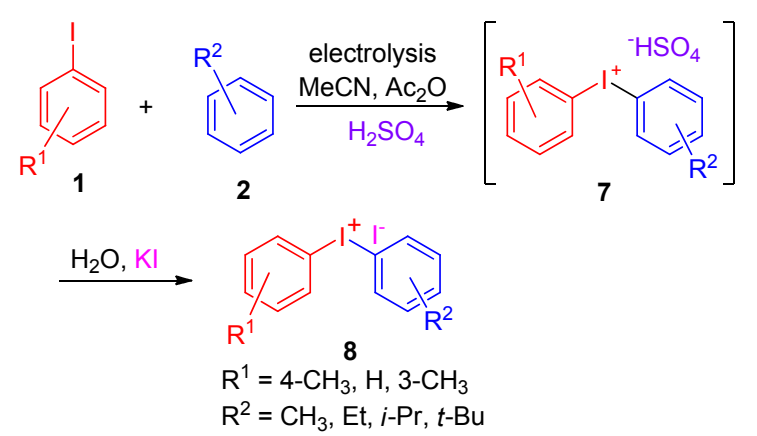

图式 2 流动化学法合成二芳基碘盐

Scheme 2 Synthesis of diaryliodonium salts in flow

\section{2 含氟高价磑试剂的电化学合成及其介导的氟 化反应}

1960 年, Schmidt 和 Meinert ${ }^{[2]}$ 首次报道了二氟碘苯 的电化学合成, 方法是以氟化银为电解质和氟源, 通过 碘苯在乙腈溶液中电解得到. 1976年, Rozhkov ${ }^{[22]}$ 指出该 实验结果不可重复, 原因是溶液的电导率太低. 1994 年, Fuchigami 等 ${ }^{[23]}$ 研究了芳基碘化物的电化学氟化, 结果 表明, 碘苯在 $\mathrm{Et}_{3} \mathrm{~N}-3 \mathrm{HF}$ 存在下的阳极氧化, 不能生成二 氟碘苯, 而是形成了碘盐 9. 若将碘苯换作 4-甲基碘苯 时, 同样也没有生成预期的二氟碘苯的产物, 而是形成 了甲基氟化产物 10. 但是在相同条件下, 用 4-硝基碘苯 作为底物时, 产物 4-硝基二氟碘苯(11d)在电解过程中逐 渐沉淀析出并通过简单的过滤得以纯化, 产率达到 53\%, 这是由于硝基官能团吸电子性能强, 致使 4-硝基碘苯 (1d)在高的电位 $(2.3 \mathrm{~V}$ vs. SCE)下发生阳极氧化; 含有强 供电子基团的 4-甲氧基碘苯(1c) 可以在较低的电位(1.9 V vs.SCE)下发生氧化, 但生成的产物 4-甲氧基二氟碘苯 (11c)的稳定性较差并且不能分离纯化(Scheme 3).

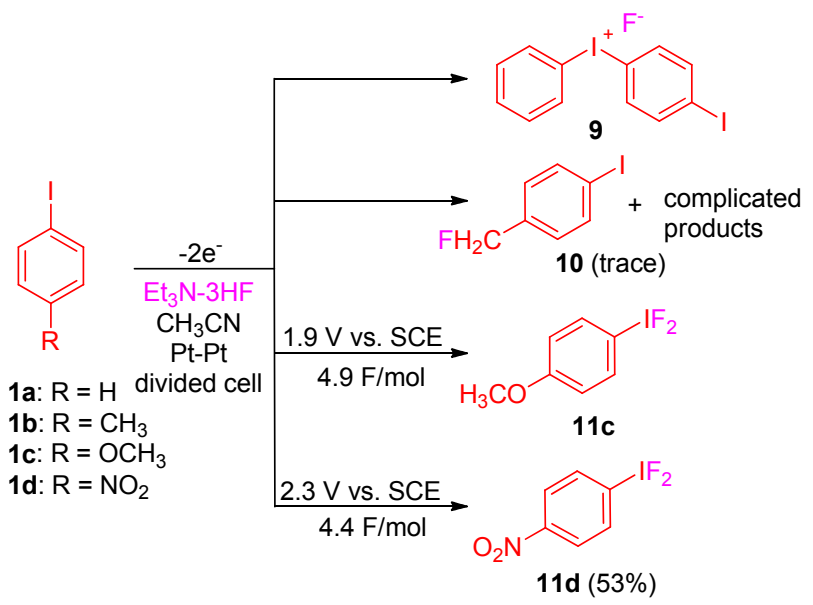

图式 3 碘苯衍生物在 $\mathrm{Et}_{3} \mathrm{~N}-3 \mathrm{HF}$ 存在下的间接阳极氧化 Scheme 3 Direct anodic oxidation of iodobenzene derivatives in $\mathrm{Et}_{3} \mathrm{~N}-3 \mathrm{HF}$
芳基碘化物高的氧化电位，成为阻碍其发生阳极氧 化的主要问题, 理论上, 这可以通过加入介质 ${ }^{[24]}$ 如氯离 子进行间接阳极氧化来解决(Scheme 4). 实验表明, 当 加入氯离子时，反应能够顺利进行，但没有生成二氟碘 苯 11，而是生成了氯化氟化的三价碘化合物 $\mathbf{1 3}^{[25]}$. 高价 碘化合物 12 和 13 可以促进二硫代缩醛的间接阳极氟化, 氟化既可用槽外法，也可用槽内法进行，并且槽内法的 氟化反应仅需催化量的芳基碘即可，例如，当加入 $5 \%$ 的 4-甲氧基碘苯时, 就能高产率地生成双氟代产物 15 (Scheme 5).

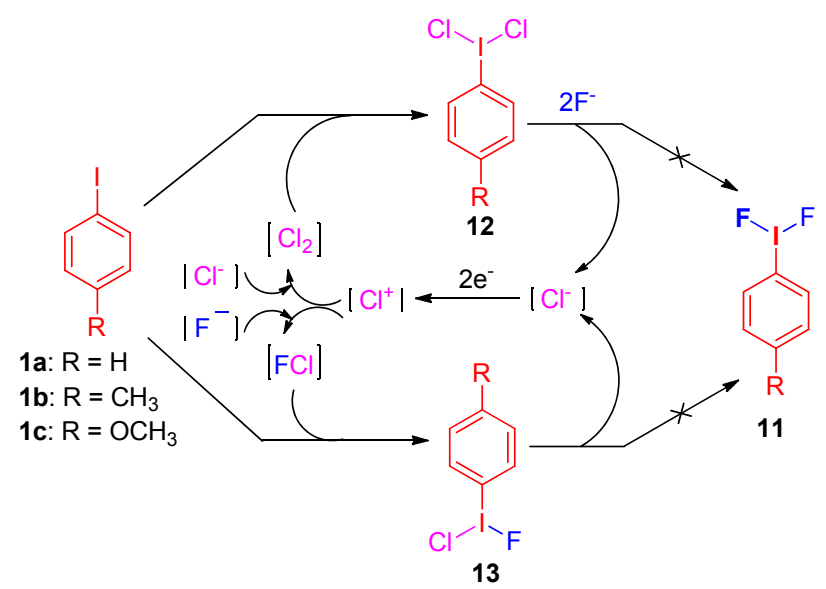

图式 4 氯离子参与的芳基碘的间接阳极氧化

Scheme 4 Indirect anodic oxidation of aryl iodine mediated by chloride ions

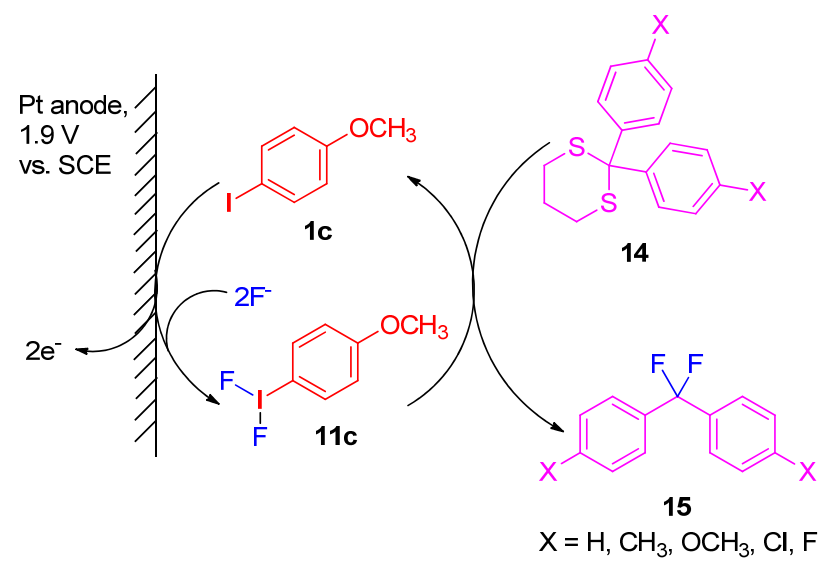

图式 5 二硫代缩醛衍生物的双氟化反应机理

Scheme 5 Mechanism of difluorination of dithioketals

1998 年, Hara 等 ${ }^{[26]}$ 发现了在羰基化合物的电化学氧 化中, 电解质 $\mathrm{Et}_{3} \mathrm{~N}-5 \mathrm{HF}$ 性能要优于 $\mathrm{Et}_{3} \mathrm{~N}-3 \mathrm{HF}$, 并且以 $\mathrm{Et}_{3} \mathrm{~N}-5 \mathrm{HF}$ 为电解质, 合成了高效的氟化试剂 4-甲基二 氟碘苯(11b)，并用于 $\beta$-二羰基化合物的间接阳极氟化. 方法是将 4-碘甲苯(1b)和 $\beta$-二羰基化合物 16 按 $1: 1$ 混 合, 置于无隔膜电解槽中恒电位 $\left(1.5 \mathrm{~V} v \mathrm{vs} . \mathrm{Ag} / \mathrm{Ag}^{+}\right)$电解, 以 50\% 79\%的产率得到 $\alpha$-氟代的 $\beta$-二羰基化合物 17 
(Eq. 4). 结果表明, $\alpha$ 位不含取代基的 $\beta$-酮酯类化合物的 氟化具有较高的选择性, 主要生成单氟化的产物 $(\mathbf{1 7} \mathbf{a} \sim$ 17d)； $\alpha$ 位不含取代基的 $\beta$-二酮类化合物的氟化选择性 较差, 除了生成单氟化产物之外,也能生成双氟化的产 物, 例如化合物 16f，除了以 50\%的产率生成单氟化产 物 $17 \mathrm{f}$ 外，也以 $6 \%$ 的产率生成 $\alpha$ 位双氟化的产物.

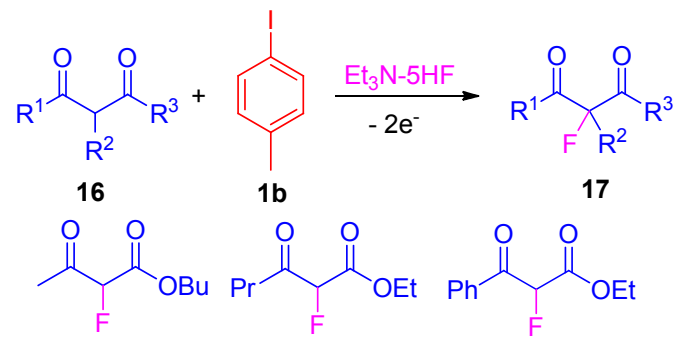

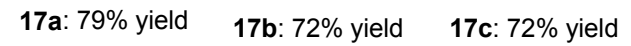<smiles>CCOC(=O)C(F)C(=O)C(F)C(=O)C(F)C(=O)C(F)(F)C(C)=O</smiles>

17d: $70 \%$ yield $\quad 17 e: 56 \%$ yield $\quad 17 f: 50 \%$ yield<smiles>CCC(=O)C(C)(C(=O)CC)C(=O)C(C)C(=O)C(C)(F)C(=O)C(C)C</smiles>

2019 年, Waldvogel 小组 ${ }^{[27]}$ 将 11b 用于烯丙基酰胺 (18)的氟化环化反应中, 生成了 5-氟甲基-2-噁唑啉类化 合物 21. 反应机理为: 首先, 4-碘甲苯(1b)在阳极发生氧 化生成 11b, 接着受到 18 双键的亲核进攻生成碘鎓离子 中间体 19, 随后在羰基的作用下发生开环生成三价碘 中间体 20, 最后, 在亲核试剂 $\mathrm{F}^{-}$的进攻下发生 $\mathrm{S}_{\mathrm{N}} 2$ 取代 生成目标产物 21 (Scheme 6).

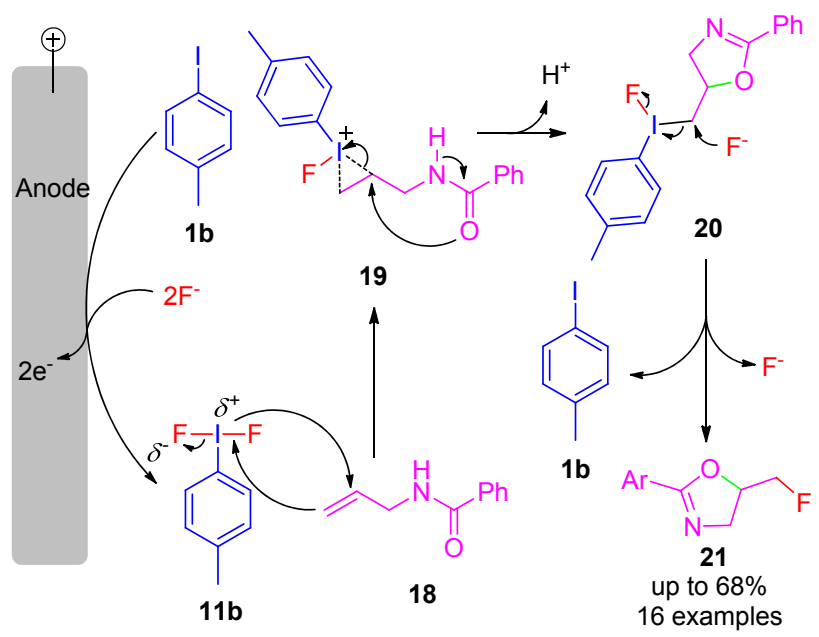

图式 $6 \mathrm{~N}$-烯丙基酰胺的氟化环化反应机理 Scheme 6 Mechanism for the fluorocyclization of $N$-allylcarboxamides

可回收循环利用的聚苯乙烯负载的碘苯和功能化
离子液体介导的选择性电催化氟化亦有报道 ${ }^{[28]}$. 2010 年, Fuchigami 小组 ${ }^{[28 a]}$ 利用聚苯乙烯负载的碘苯 23 实现 了有机硫化合物黄原酸盐 22、糖衍生物 25 、碳酸乙烯 酯 27 的间接阳极氟化，高产率地生成氟化产物 24、26 和 28. 反应是在装有 $\mathrm{Et}_{3} \mathrm{~N}-5 \mathrm{HF}$ 和 $\mathrm{Et}_{4} \mathrm{NCl}$ 混合液的无隔 膜电解槽中恒流 $\left(5.0 \mathrm{~mA} / \mathrm{cm}^{2}\right)$ 条件下电解完成(Scheme 7). 聚苯乙烯负载的碘苯 23 经过滤可从电解液中分离, 并且循环 10 次之余，氟化产率仍能高达 $80 \%$ 左右. 反应 机理如 Scheme 8 所示, 首先, $\mathrm{Cl}^{-}$在阳极发生氧化生成 $\mathrm{Cl}^{+}$, 接着与 23 反应生成 $\mathrm{PhI}^{+} \mathrm{Cl}$, 随后 $\mathrm{PhI}^{+} \mathrm{Cl}$ 捕获 $\mathrm{F}^{-}$ 得到三价碘试剂 $\mathbf{3 0}$, 最后, $\mathbf{3 0}$ 作为有机硫化物的氧化剂 进行氧化氟化得到氟化产物.

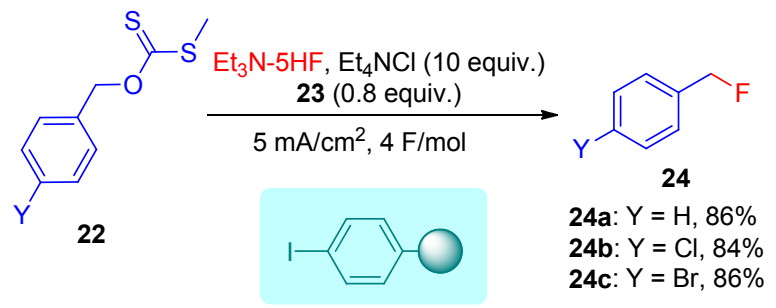

23
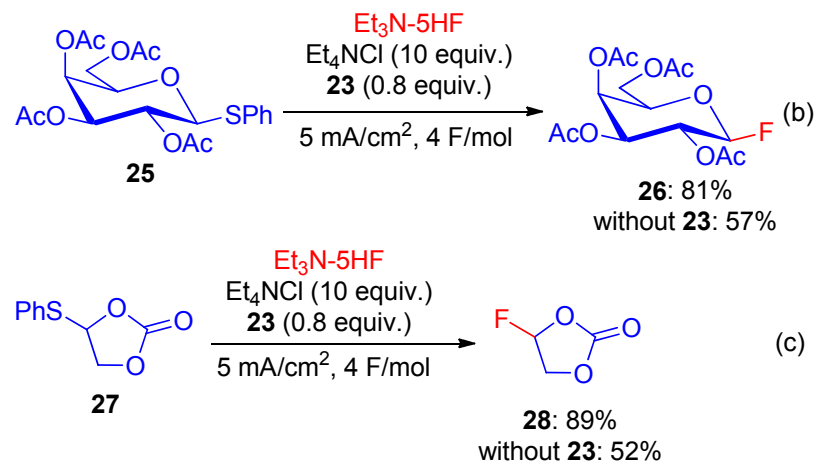

图式 7 有机硫化合物的间接阳极氟化

Scheme 7 Indirect anodic fluorination of organosulfur compounds

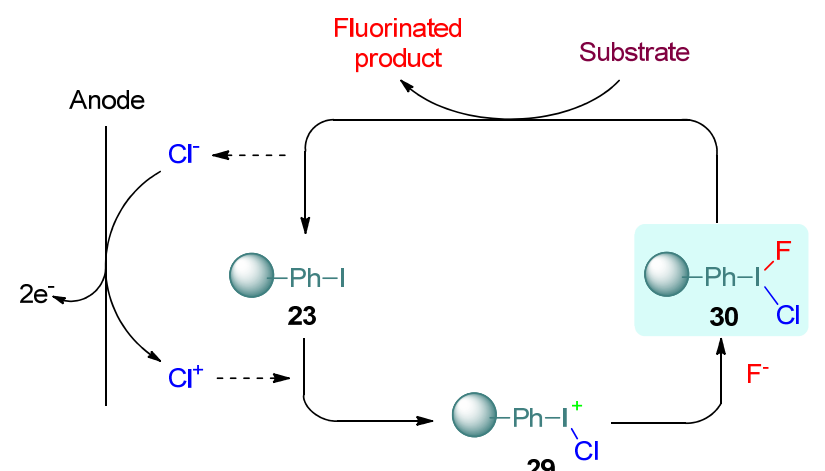

图式 8 聚苯乙烯负载的碘苯介导的间接阳极氟化机理 Scheme 8 Mechanism of indirect anodic fluorination mediated by polystyrene-supported iodobenzene

在功能化离子液体 31 介导的间接阳极氟化中, 反 
应产物可通过有机溶剂如乙醚等萃取分离, 功能化的离 子液体可高效回收循环利用(图 1). 伏安法测得 31 在 $\mathrm{Et}_{4} \mathrm{NF}-4 \mathrm{HF} / \mathrm{MeCN}$ 中的氧化电位为 $E_{1 / 2}=1.91 \mathrm{~V}$ (vs. $\mathrm{SCE}$ ), 与简单芳基碘化物的氧化电位相当, 因此, 很多 底物如 2-嘧啶硫化物 32 以及具有活性苄位的化合物 34 等都能在装有铂电极无隔膜电解槽中恒电流 (5.0 $\mathrm{mA} / \mathrm{cm}^{2}$ )条件下高效氟化(Scheme 9) ${ }^{[28 \mathrm{~b}]}$.

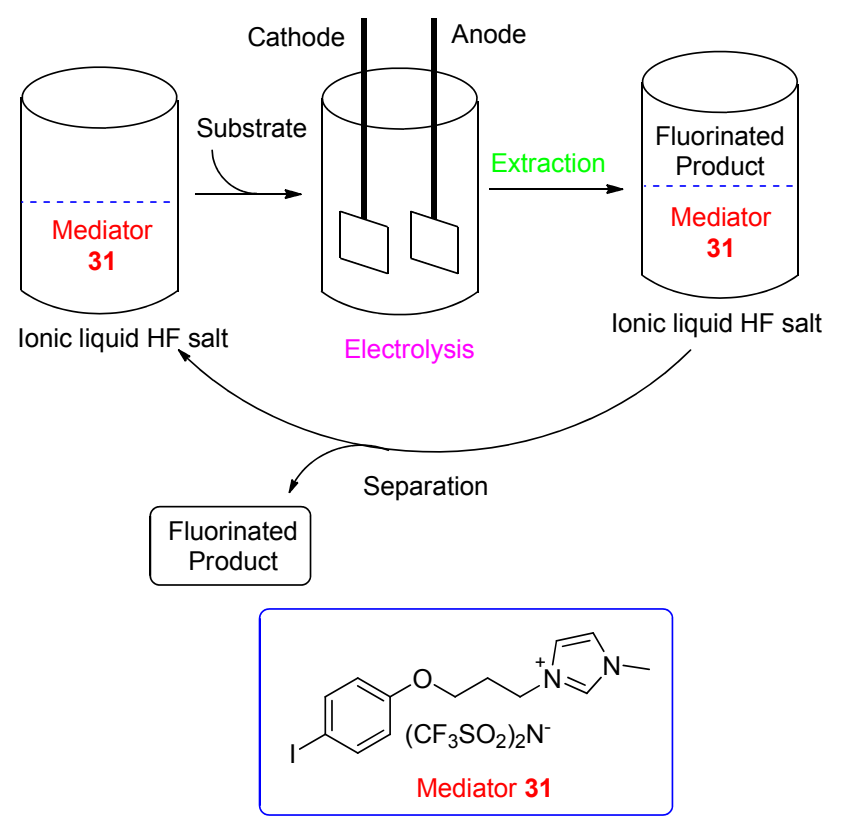

图 1 利用 31 进行的间接阳极氟化

Figure 1 Use of $\mathbf{3 1}$ for indirect anodic fluorination

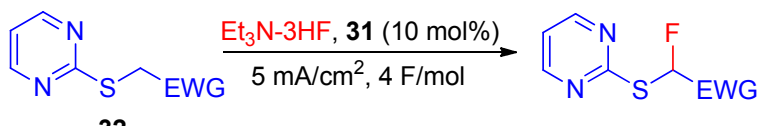

32

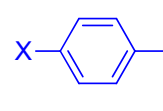

34

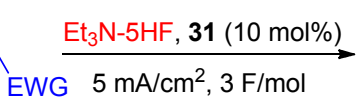

33a: $\mathrm{EWG}=\mathrm{CO}_{2} \mathrm{Et}, 87 \%$ 33b: $E W G=C N, 72 \%$

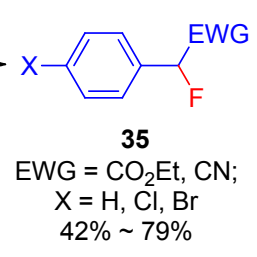

图式 92 -嘧啶硫化物和茮位的间接阳极氟化

Scheme 9 Indirect anodic fluorination of 2-pyrimidylsulfides and benzylic positions

\section{3 双(三氟乙氧基)磑苯的电化学合成及其介导 的氧化环化}

2006 年, Nishiyama 小组 ${ }^{[29 a]}$ 报道了高价碘试剂双(三 氟乙氧基)碘苯 36 的电化学合成(图 2), 并成功将其用于 天然黄原酮衍生物芒果苷的氧化 ${ }^{[29 b]}$ 及各种含杂原子的 有机分子和天然产物 ${ }^{[29]}$ 的合成之中.

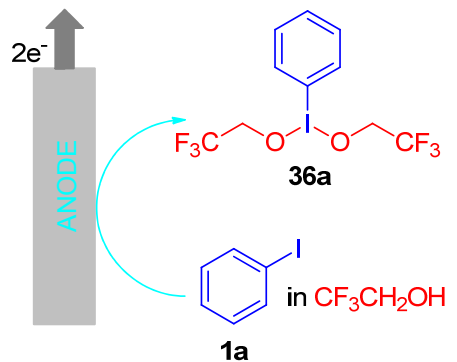

$$
\begin{aligned}
& \text { Condition } \\
& \text { Anode: Glassy carbon } \\
& \text { Cathode: Platinum wire } \\
& \text { Current: } 0.3 \mathrm{~mA} / \mathrm{cm}^{2} \\
& \text { Supporting salt: } 0.5 \mathrm{~mol} / \mathrm{L} \mathrm{LiClO}_{4}
\end{aligned}
$$

图 2 电化学合成高价碘试剂 36

Figure 2 Electrochemical generation of the hypervalent iodine reagent 36

将芳基碘化物阳极氧化生成 36, 不经分离, 直接用 于酚类化合物的氧化螺环化，反应是将芳基碘置于由玻 璃碳烧杯作为阳极、铂丝作为阴极的无隔膜电解槽中, 恒电流 $\left(0.3 \mathrm{~mA} / \mathrm{cm}^{2}\right)$ 条件下, 用含 $\mathrm{LiClO}_{4}(0.05 \mathrm{~mol} / \mathrm{L})$ 的 三氟乙醇作为支撑电解质进行电解，除 4-硝基碘苯，其 电解产物能够分离纯化为稳定的固体之外, 其它芳基碘 化物电解生成的高价碘试剂只能在溶液中稳定存在. 实 验表明，电化学合成的三价碘试剂 36, 其氧化性能优于 双三氟乙酸碘苯，在酚类化合物的螺环化反应中，能以 几乎定量的产率(高达 97\%)生成目标产物 38 (Scheme $10)^{[30 a]}$.

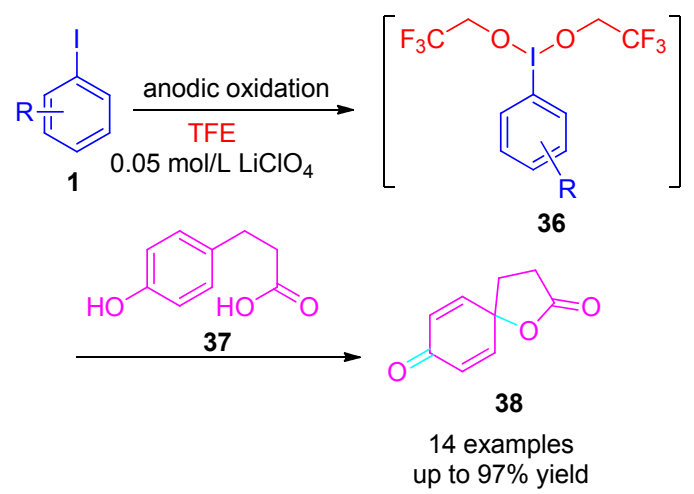

图式 10 碘苯衍生物 $\mathbf{1}$ 的阳极氧化及利用 $\mathbf{3 6}$ 进行酚的氧化 Scheme 10 Anodic oxidation of iodobenzenes 1 and use of $\mathbf{3 6}$ in phenolic oxidation

此外, 电化学合成的高价碘试剂 36 还可用于甲氧 基酰胺衍生物 39 的脱芳构化/螺内酰胺化反应，高产率 地得到产物 40 (Eq. 5) ${ }^{[29 a]}$. 实验结果表明, 当甲氧基酰 胺衍生物 39 的取代基 $\mathrm{R}$ 为氢或卤素时, 生成唯一的螺 环产物 40; 然而, 当取代基 $\mathrm{R}$ 为供电子基团如甲氧基 时, 反应没有选择性, 将以大约 $1: 1$ 的比例得到 40 和 41 的混合物. 另一方面, 苯环中甲氧基的位置也影响反 
应的结果，例如当甲氧基和酰胺处于间位时，生成甲氧 基对位亲电进攻的产物喹啉酮衍生物 43 要多于邻位环 化产物 44(比例为 $6.5: 1$ ); 当甲氧基和酰胺处于邻位时, 将以 $82 \%$ 的产率生成唯一的螺环产物 46 (Scheme 11).<smiles>[R]c1cc(CCC(=O)NOC)ccc1OC</smiles>

\begin{tabular}{llll}
\hline & $\mathrm{R}=\mathrm{H}$ & $\mathrm{R}=\mathrm{Cl}$ & $\mathrm{R}=\mathrm{Br}$ \\
Direct anodic oxidation & $67 \%$ & $45 \%$ & $59 \%$ \\
[Bis(trifluoroacetoxy)iodo]benzene & quant. & $67 \%$ & $75 \%$ \\
Electrogenerated 36a & quant. & $94 \%$ & $89 \%$ \\
\hline
\end{tabular}

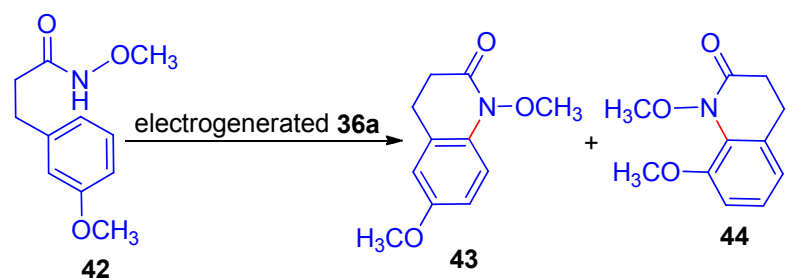<smiles>CONC(=O)CCc1ccccc1OC</smiles>

图式 11 甲氧基酰胺衍生物 $\mathbf{4 2}$ 和 $\mathbf{4 5}$ 的氧化

Scheme 11 Oxidation of the methoxyamide derivatives $\mathbf{4 2}$ and 45

为提高邻位环化产物的产率, Nishiyama 等 ${ }^{[30 b]}$ 将酰 胺衍生物苯环中甲氧基的对位用 $\mathrm{Cl}, \mathrm{OAc}, \mathrm{Br}, \mathrm{CN}$ 取代, 结果表明, $\mathrm{CN}$ 取代的酰胺发生了邻位的环化, 以 $17 \%$ 的 产率得到预期产物 51d, $\mathrm{Cl}, \mathrm{OAc}, \mathrm{Br}$ 取代的酰胺则发生 对位环化, 高产率地生成喹啉酮类化合物 50, 反应机理 见 Scheme 12. 作者又将酰胺衍生物苯环中 $\mathrm{OCH}_{3}$ 换作 $\mathrm{BnO}$ 和 $\mathrm{AcO}$, 前者以较高的产率生成 50, 而后者生成了 复杂的混合物.

上述喹啉酮类化合物的合成方法曾被成功地用于

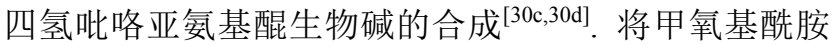
衍生物 52 通过电化学合成的三价碘试剂 36a 的氧化环 化, 以 $62 \%$ 的产率生成喹啉酮化合物 $\mathbf{5 3}$, 随后, 经过 5 步反应, 最终以 $31 \%$ 的产率得到生物碱 Damirone 和 Makaluvamine 的重要中间体 $\alpha$-二酮 54 (Scheme 13) ${ }^{[30 \mathrm{e}]}$.

$N$-乙酰色胺 $(55)$ 在电化学合成的 $36 \mathrm{a}$ 或醋酸碘苯作 用下发生氧化环化, 分别以 $30 \%$ 和 $38 \%$ 的产率合成了吡

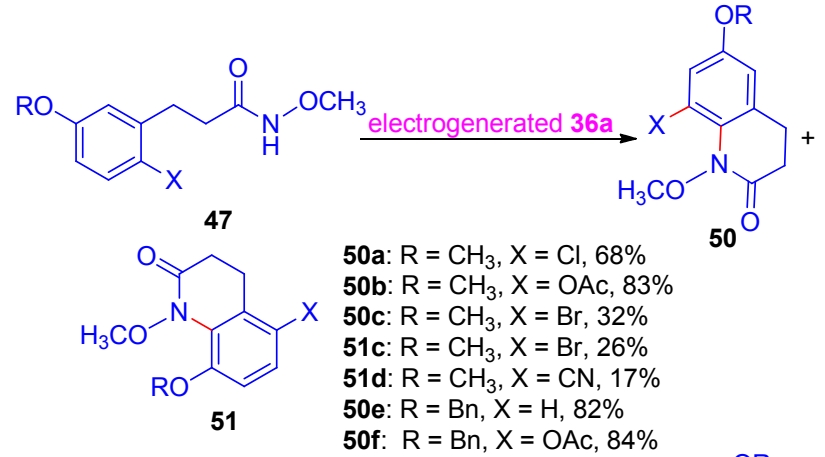

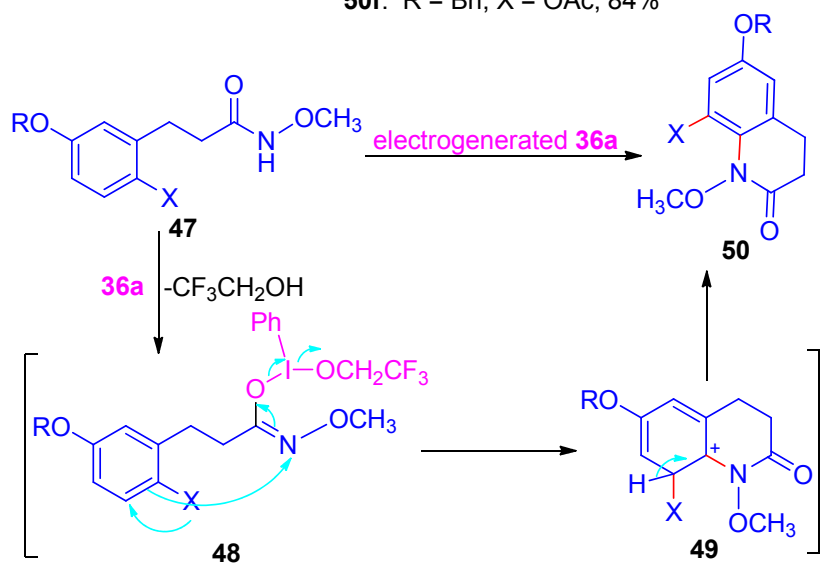

图式 12 化合物 $\mathbf{4 7}$ 环化重排机理

Scheme 12 Mechanism of the cyclization and rearrangement of compound $\mathbf{4 7}$

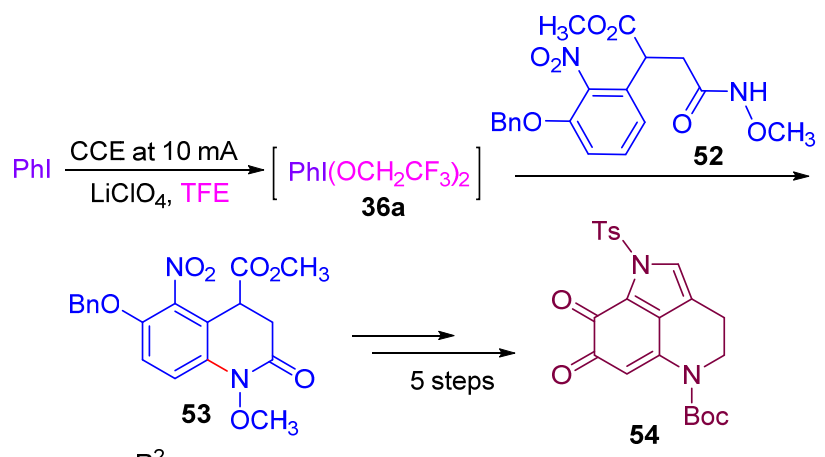<smiles>[R]N1CCc2cn([R])c3c2C1=CC(=O)C3=O</smiles><smiles>NC1=CC2=NCCc3c[nH]c(c32)C1=O</smiles>

Damirone $A: R^{1}=R^{2}=M e$ Damirone $B: R^{1}=M e, R^{2}=H$ Damirone C: $\mathrm{R}^{1}=\mathrm{R}^{2}=\mathrm{H}$<smiles>CC(C)CNC1=CC2=NCCc3c[nH]c(c32)C1=O</smiles>

Makaluvamine

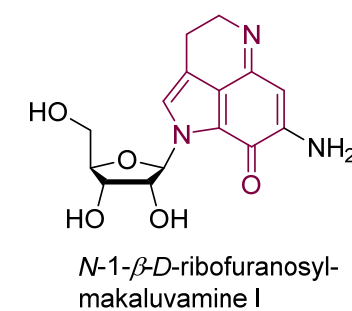

图式 $13 \alpha$ 二酮 54 的合成及四氢吡咯亚氨基醌生物碱的结构 Scheme 13 Synthesis of the $\alpha$-diketone 54 and the stucture of tetrahydropyrroloiminoquinone alkaloids 
咯并吲哚的衍生物 56a 和 56b. 以 56b 为起始原料, 经 过三步反应，将以 $28 \%$ 和 $35 \%$ 的总收率得到天然产物 CPC-1 和 Debromofrustaminol B (Eq. 6) ${ }^{[30 f]}$.

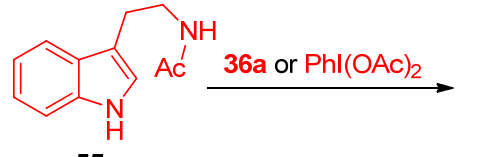

55

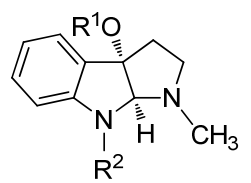

CPC-1: $\mathrm{R}^{1}=\mathrm{R}^{2}=\mathrm{CH}_{3}$;

Debromofrustaminol $B: R^{1}=H, R^{2}=$ prenyl

苯乙酰苯胺衍生物 57 也可在电化学合成的三价碘 试剂 36a 的作用下发生氧化环化反应 ${ }^{[30 \mathrm{~g}]}$ ，以 $0 \sim 91 \%$ 的 产率生成咔唑类化合物(Eq. 7). 然而, 当底物 $\mathbf{5 7}$ 中的取 代基 $\mathrm{R}^{3}$ 为甲氧基时, 仅以 $42 \%$ 的产率生成目标产物 (58c); 另外, 酰胺官能团对位取代基 $\mathrm{R}^{4}$ 无论是吸电子 的硝基还是供电子的甲氧基，都不能生成目标产物; 其 它取代基的底物都能以较好的产率发生环化生成咔唑 类化合物 $\mathbf{5 8 a} \sim \mathbf{5 8 f}$ ，作者还利用该方法合成了抗菌剂甘 唑啉.
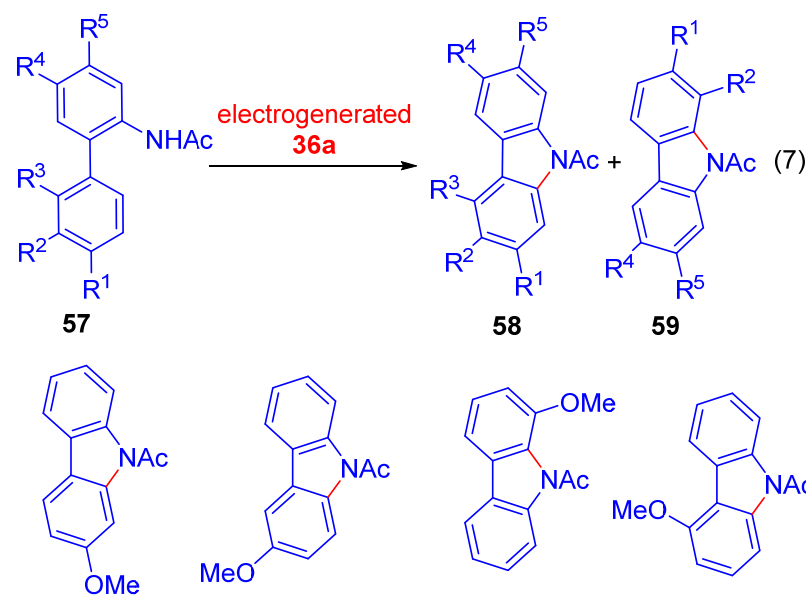

58a: $91 \%$ yield $\quad$ 58b: $40 \%$ yield
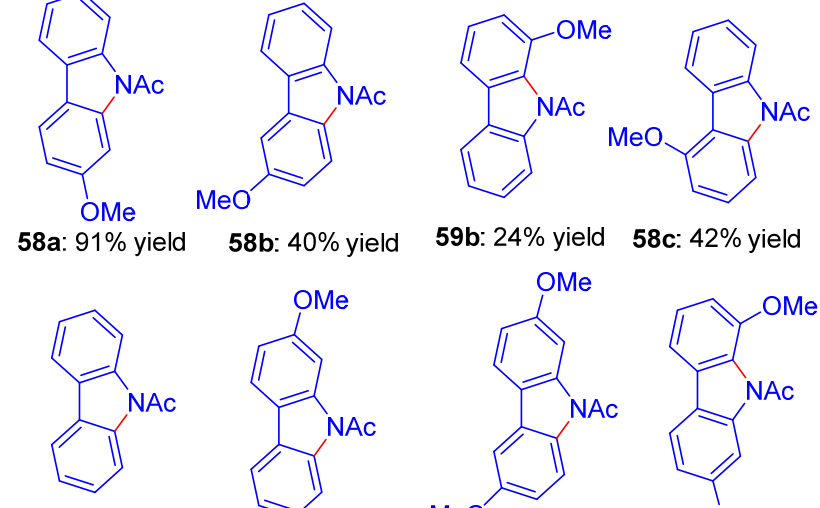

58d: $73 \%$ yield

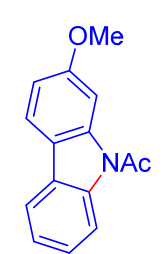

58e: $83 \%$ yield

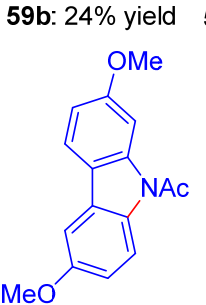

58f: $44 \%$ yield<smiles>COc1ccc2c3cccc(OC)c3n(C)c2c1</smiles>

59f: $24 \%$ yield
液和 $0.8 \mathrm{~mol} / \mathrm{L}$ 高氯酸锂的 $\mathrm{H}$-型电解池中, 分别将碘苯 和乙烯基苯甲酸酯加入到阳极室，室温、恒电流密度 $\left(7.5 \mathrm{~mA} / \mathrm{cm}^{2}\right)$ 下电解, 将以较高的产率生成目标产物异 色满酮类化合物. 实验结果表明，苯环取代基的电子效 应对反应有一定的影响, 例如, 当 $\mathrm{R}^{1}$ 为氢或吸电子基团 时，反应能够顺利进行，当 $\mathrm{R}^{1}$ 为供电子基团如甲氧基时 (61f), 产物稳定性差, 会发生分解; 芳环取代基的位阻 效应对反应影响不大. 然而双键位取代基的位阻对产率 和 $d r$ 值有一定的影响，例如，当取代基为立体位阻大的 环己基时(611), 其 $d r$ 值高达 $90: 10$, 但其产率较低, 仅 有 $45 \%$, 当取代基为立体位阻小的正丙基时 $(61 \mathbf{j})$, 虽然 产率能达到 $60 \%$, 但是 $d r$ 值很低，只有 $64: 36$.

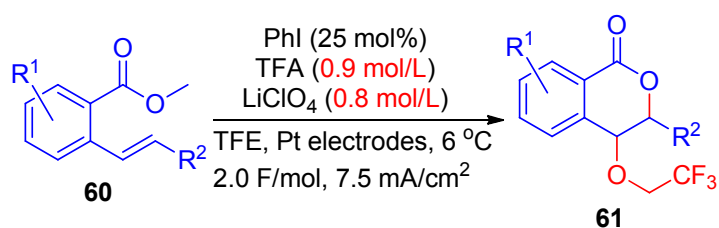<smiles>O=C1OCC(OCC(F)(F)F)c2ccc(F)cc21</smiles>

61a: $59 \%$ yieid<smiles>O=C1OCC(OCC(F)(F)F)c2ccccc21</smiles>

61b: $58 \%$ yieid<smiles>O=C1OCC(OCC(F)(F)F)c2ccc(Cl)cc21</smiles>

61c: $54 \%$ yieid<smiles>O=C1OCC(OCC(F)(F)F)c2cc(Br)ccc21</smiles>

61d: $57 \%$ yieid<smiles>CC(F)(F)COC1COC(=O)c2cc(-c3ccccc3)ccc21</smiles>

61e: $45 \%$ yieid<smiles>O=C1OCC(OCC(F)(F)F)c2ccc(O)cc21</smiles>

61f: trace yieid<smiles>CC(=O)c1ccc2c(c1)C(=O)OCC2OCC(F)(F)F</smiles>

61g: $54 \%$ yieid<smiles>Cc1cccc2cc3c(cc12)C(OCC(F)(F)F)COC3=O</smiles>

61h: $54 \%$ yieid<smiles>CCCC1OC(=O)c2cc(F)ccc2C1OCC(F)(F)F</smiles>

61i: $60 \%$ yieid
Möckel 等 ${ }^{[31]}$ 报道了电化学产生的三价碘试剂介导 的乙烯基苯甲酸酯 60 的内酯化反应(Eq. 8), 合成了异色 满酮类化合物 61 , 并将三氟乙氧基引入到产物分子中. 反应是在装有 $0.9 \mathrm{~mol} / \mathrm{L}$ 三氟乙酸的 $10 \mathrm{~mL}$ 三氟乙醇溶

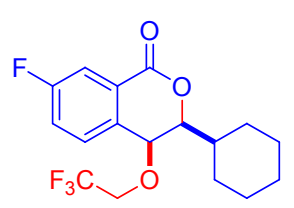

61I: $45 \%$ yieid $d r=90: 10$ 61j: $60 \%$ yieid

61k: $43 \%$ yieid $d r=74: 26$
2016 年, Francke 小组 ${ }^{[32 \mathrm{a}]}$ 报道了一种新型可回收循 环利用的一价碘/三价碘氧化还原介质, 并成功实现了 
$\mathrm{C}-\mathrm{N}$ 和 $\mathrm{C}-\mathrm{C}$ 键的直接氧化偶联. 在一价碘化合物 63 中，4-碘苯部分充当了氧化还原活性单元，烷基铵盐既 能提供导电的离子, 又能便于产物和电解质的分离, 还 能回收利用. 其合成方法是以碘苯 $1 \mathrm{a}$ 为原料, 经三步反 应, 以 74\%的总收率得到 63 (Scheme 14). 一价碘化物 铵盐 63 在氟代醇溶剂如三氟乙醇和 $1,1,1,3,3,3$-六氟异 丙醇中发生阳极氧化, 生成相应的高价碘试剂 64(图 3), 用于各种氧化偶联反应当中, 氟代醇溶剂的选择是基于 其具有优良的电化学特性, 如高的导电率和良好的稳定 性, 同时还可以稳定产生的三价碘试剂 64, 实验表明, 64b 在六氟异丙醇中可稳定存在, 然而若将六氟异丙醇 移除, 64b 便会逐渐分解.
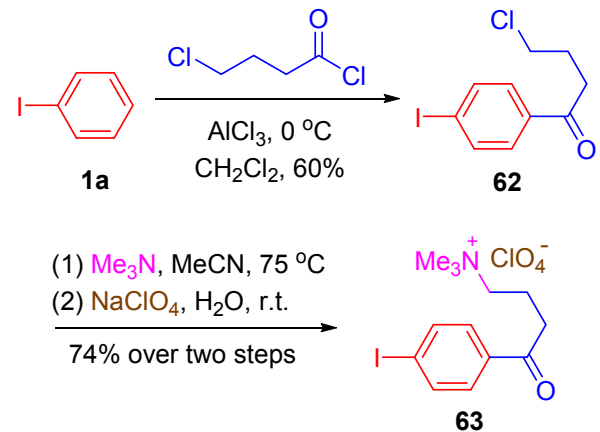

图式 14 支撑电解质的 63 的合成

Scheme 14 Synthesis of supporting electrolyte 63

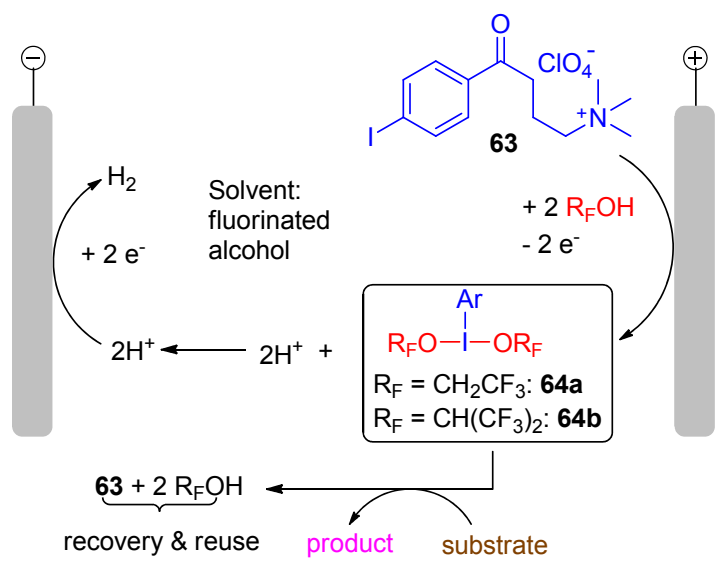

图 3 一价碘/三价碘氧化还原电对作为电合成的介质体系

Figure 3 Iodine(I)/iodine(III) redox couple as a mediatory system for electrosynthesis

2017 年, Francke 小组 ${ }^{[32 b]}$ 又将高价碘试剂 64b 用于 2-(亚芳基氨基)苯酚(65)的氧化环化反应之中 (Scheme $15)$, 以 $40 \% \sim 95 \%$ 的产率合成系列苯并噁唑类化合物 66, 这种槽外法电化学合成苯并噁唑类化合物的方法具 有官能团适应性广泛等优点, 例如含双键、溴、羧酸的 底物均适应于该反应.

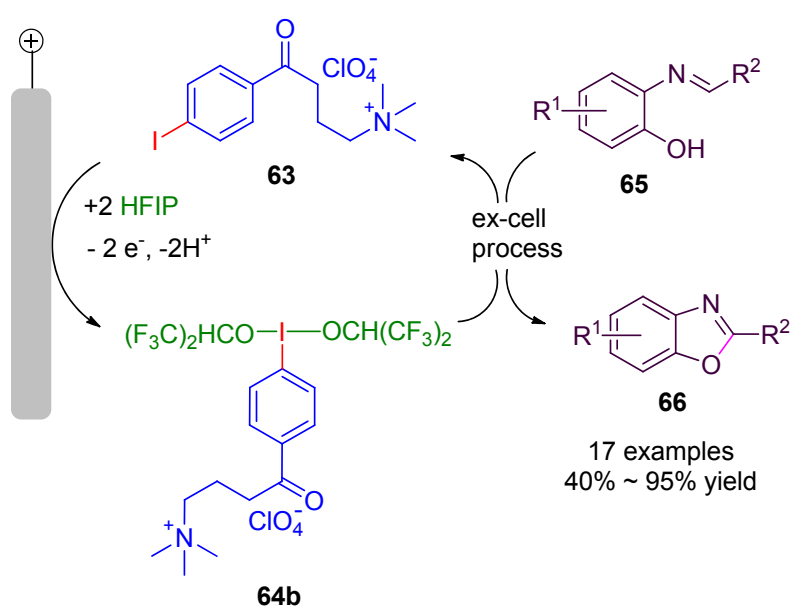

图式 15 间接电化学合成苯并噁唑类化合物 66

Scheme 15 Indirect electrolysis of benzoxazoles 66

\section{4 结论和展望}

综述了阳极氧化芳基碘化物制备高价碘试剂的电 化学合成方法及其应用. 无论是在隔膜还是无隔膜电解 槽中，芳基碘化物的阳极氧化都能在恒电流、恒电位条 件下成功实现. 利用绿色的电化学技术合成高价碘试 剂, 避免了使用化学氧化剂, 减少污染, 节约资源, 并 且电化学合成的高价碘试剂, 反应性能与商业化试剂如 醋酸碘苯相当，甚至有时高于醋酸碘苯，因此，无论采 用槽外法还是槽内法，都可以促进 $\beta$-二羰基等化合物的 氟化及甲氧基酰胺等化合物的氧化环化，还可以成功地 用于 Debromofrustaminol B 等天然产物的全合成. 另外, 一价碘/三价碘氧化还原介质介导的碳碳键、碳氮等化学 键的形成反应已有较广泛的研究, 并证明其性能高效; 同时，可回收的芳基碘介质也可用于氧化环化反应中. 尽管高价碘试剂的电化学合成及应用目前已有深入地 研究, 然而仍具有一定的局限性, 这使得该领域的研究 将具有更广阔的前景.

\section{References}

[1] (a) "Hypervalent Iodine Chemistry: Modern Developments in Organic Synthesis" in Topics in Current Chemistry, Vol. 373, Ed.: Wirth, T., Springer-Verlag, Switzerland, 2016,

(b) Zhdankin, V. V.; Stang, P. J. Chem. Rev. 2008, 108, 5299.

(c) Charpentier, J.; Früh, N.; Togni, A. Chem. Rev. 2015, 115, 650.

(d) Yoshimura, A.; Zhdankin, V. V. Chem. Rev. 2016, 116, 3328.

(e) Duan, Y.-N.; Jiang, S.; Han, Y.-C.; Sun, B.; Zhang, C. Chin. J. Org. Chem. 2016, 36, 1973 (in Chinese).

(段亚南, 姜山, 韩永超, 孙博, 张弛, 有机化学, 2016, 36, 1973.)

(f) Zhang, H.; Tang, R.; Wu, J.; Hu, Y. Chemistry 2018, 681 (in Chinese).

(张怀远, 唐蓉萍, 伍家卫, 胡雨来, 化学通报, 2018, 681.)

(g) Ma, J.; Chen, L.; Yuan, Z.; Cheng, H. Chin. J. Org. Chem. 2018, 38,1586 (in Chinese).

(马姣丽, 陈立成, 袁中文, 程辉成, 有机化学, 2018, 38, 1586.)

(h) Yan, Y.; Cui, C.; Li, Z. Chin. J. Org. Chem. 2018, 38, 2501 (in Chinese). 
(问溢哲，崔畅，李政，有机化学，2018, 38, 2501.)

[2] (a) Muñiz, K.; Barreiro, L.; Romero, R. M.; Martínez, C. J. Am. Chem. Soc. 2017, 139, 4354.

(b) Fujita, M.; Miura, K.; Sugimura, T. Beilstein J. Org. Chem. 2018, 14, 659

(c) Banik, S. M.; Mennie, K. M.; Jacobsen, E. N. J. Am. Chem. Soc. 2017, 139, 9152.

[3] Smith, D. C.; Vitaku, E.; Njardarson, J. T. Org. Lett. 2017, 19, 3508.

[4] Hori, M.; Guo, J.-D.; Yanagi, T.; Nogi, K.; Sasamori, T.; Yorimitsu, H. Angew. Chem., Int. Ed. 2018, 57, 4663.

[5] (a) Zhang, H.; Huang, D.; Wang, K.-H.; Li, J.; Su, Y.; Hu. Y. J. Org. Chem. 2017, 82, 1600 .

(b) Chi, Y.; Zhang, W.-X.; Xi, Z. Org. Lett. 2014, 16, 6274

(c) Chi, Y.; Yan, H.; Zhang, W.-X.; Xi, Z. Chem.-Eur. J. 2017, 23, 757.

(d) Chi, Y.; Yan, H.; Zhang, W.-X.; Xi, Z. Org. Lett. 2017, 19, 2694.

(e) Alazet, S.; Vaillant, F. L.; Nicolai, S.; Courant, T.; Waser, J. Chem.-Eur. J. 2017, 23, 9501.

(f) Colomer, I.; Batchelor-McAuley, C.; Odell, B.; Donohoe, T. J.; Compton, R. G. J. Am. Chem. Soc. 2016, 138, 8855.

(g) Shen, H.; Deng, Q.; Liu, R.; Feng, Y.; Zheng, C.; Xiong, Y. Org. Chem. Front. 2017, 4, 1806.

(h) Wang, Z.; Zhong, J.; Zheng, C.; Fan, R. Org. Chem. Front. 2017, 4, 1005 .

[6] Zhang, H.; Huang, D.; Wang, K.-H.; Li, J.; Su, Y.; Hu, Y. Org. Biomol. Chem. 2017, 15, 5337.

[7] Pluta, R.; Krach, P. E.; Cavallo, L.; Falivene, L.; Rueping, M. ACS Catal. 2018, 8, 2582.

[8] Zhang, H.; Wang, K.-H.; Wang, J.; Su, Y.; Huang, D.; Hu, Y. Org. Biomol. Chem. 2019, 17, 2940.

[9] Brown, M.; Kumar, R.; Rehbein, J.; Wirth, T. Chem.-Eur. J. 2016, $22,4030$.

[10] Haubenreisser, S.; Wöste, T. H.; Martínez, C.; Ishihara, K.; Muñiz, K. Angew. Chem., Int. Ed. 2016, 55, 413.

[11] Martínez, C.; Bosnidou, A. E.; Allmendinger, S.; Muñiz, K. Chem.Eur. J. 2016, 22, 9929.

[12] Zhdankin, V. V. Hypervalent Iodine Chemistry: Preparation, Structure, and Synthetic Applications of Polyvalent Iodine Compounds, John Wiley \& Sons, Chichester, UK, 2013, pp. 21 143.

[13] (a) Yang, Q.-L.; Wang, X.-Y.; Lu, J.-Y.; Zhang, L.-P.; Fang, P.; Mei, T.-S. J.Am. Chem. Soc. 2018, 140, 11487.

(b) Xiong, P.; Xu, H.-H.; Song, J.; Xu, H.-C. J. Am. Chem. Soc. 2018, 140, 2460.

(c) Yan, M.; Kawamata, Y.; Baran, P. S. Angew. Chem., Int. Ed. 2018, 57, 4149.

(d) Möhle, S.; Zirbes, M.; Rodrigo, E.; Gieshoff, T.; Wiebe, A.; Waldvogel, S. R. Angew. Chem., Int. Ed. 2018, 57, 6018.

(e) Elsherbini, M.; Wirth, T. Chem.-Eur. J. 2018, 24, 13399.

(f) Zhang, Z.; Zhang, L.; Cao, Y.; Li, F.; Bai, G.; Liu, G.; Yang, Y.; Mo, F. Org. Lett. 2019, 21, 762.

(g) Chang, X.; Zhang, Q.; Guo, C. Org. Lett. 2019, $21,10$.

(h) Lian, F.; Sun, C.; Xu, K.; Zeng, C. Org. Lett. 2019, 21, 156.

[14] Stuart, D. R. Synlett 2017, 28, 275.

[15] (a) Bielawski, M.; Olofsson, B. Chem. Commun. 2007, 2521.

(b) Bielawski, M.; Zhu, M.; Olofsson, B. Adv. Synth. Catal. 2007, $349,2610$. (c) Bielawski, M.; Aili, D.; Olofsson, B. J. Org. Chem. 2008, 73, 4602.

(d) Merritt, E. A.; Olofsson, B. Angew. Chem., Int. Ed. 2009, 48, 9052.

(e) Jalalian, N.; Olofsson, B. Tetrahedron 2010, 66, 5793.

(f) Bouma, M. J.; Olofsson, B. Chem.-Eur. J. 2012, 18, 14242.

[16] (a) Lindstedt, E.; Reitti, M.; Olofsson, B. J. Org. Chem. 2017, 82, 11909 .

(b) Laudadio, G.; Gemoets, H. P. L.; Hessel, V.; Noël, T. J. Org. Chem. 2017, 82, 11735.

[17] Miller, L. L.; Hoffmann, A. K. J. Am. Chem. Soc. 1967, 89, 593.

[18] Hoffelner, H.; Lorch, H. W.; Wendt, H. J. Electroanal. Chem. 1975, $66,183$.

[19] (a) Peacock, M. J.; Pletcher, D. Tetrahedron Lett. 2000, 41, 8995. (b) Peacock, M. J.; Pletcher, D. J. Electrochem. Soc. 2001, 148, D37.

[20] (a) Folgueiras-Amador, A. A.; Philipps, K.; Guilbaud, S.; Poelakker, J.; Wirth, T. Angew. Chem., Int. Ed. 2017, 56, 15446.

(b) Folgueiras-Amador, A. A.; Qian, X.-Y.; Xu, H.-C.; Wirth, T. Chem.-Eur. J. 2018, 24, 487.

(c) Pletcher, D.; Green, R. A.; Brown, R. C. D. Chem. Rev. 2018, $118,4573$.

(d) Folgueiras-Amador, A. A.; Wirth, T. J. Flow Chem. 2017, 7, 94. (e) Watts, K.; Gattrell, W.; Wirth, T. Beilstein J. Org. Chem. 2011, 7,1108 .

[21] Schmidt, H.; Meinert, H. Angew. Chem. 1960, 72, 109.

[22] Rozhkov, I. N. Russ. Chem. Rev. 1976, 45, 615.

[23] Fuchigami, T.; Fujita, T. J. Org. Chem. 1994, 59, 7190.

[24] Francke, R.; Little, R. D. Chem. Soc. Rev. 2014, 43, 2492.

[25] Fujita, T.; Fuchigami, T. Tetrahedron Lett. 1996, 37, 4725.

[26] Hara, S.; Hatakeyama, T.; Chen, S.-Q.; Ishi-i, K.; Yoshida, M.; Sawaguchi, M.; Fukuhara, T.; Yoneda, N. J. Fluorine Chem. 1998, 87,189

[27] Haupt, J. D.; Berger, M.; Waldvogel, S. R. Org. Lett. 2019, 21, 242.

[28] (a) Sawamura, T.; Kuribayashi, S.; Inagi, S.; Fuchigami, T. Adv. Synth. Catal. 2010, 352, 2757.

(b) Sawamura, T.; Kuribayashi S.; Inagi, S.; Fuchigami, T. Org. Lett. 2010, 12, 644.

[29] (a) Amano, Y.; Nishiyama, S. Tetrahedron Lett. 2006, 47, 6505.

(b) Nishihama, Y.; Amano, Y.; Ogamino, T.; Nishiyama, S. Electrochemistry 2006, 74, 609

(c) Kajiyama, D.; Saitoh, T.; Nishiyama, S. Electrochemistry 2013, 81,319 .

[30] (a) Amano, Y.; Nishiyama, S. Heterocycles 2008, 75, 1997.

(b) Amano, Y.; Inoue, K.; Nishiyama, S. Synlett 2008, 134.

(c) Izawa, T.; Nishiyama, S.; Yamamura, S. Tetrahedron 1994, 50, 13593.

(d) Faulkner, D. J. Nat. Prod. Rep. 2001, $18,1$.

(e) Inoue, K.; Ishikawa, Y.; Nishiyama, S. Org. Lett. 2010, 12, 436.

(f) Kajiyama, D.; Saitoh, T.; Yamaguchi, S.; Nishiyama, S. Synthesis 2012, 44, 1667.

(g) Kajiyama, D.; Inoue, K.; Ishikawa, Y.; Nishiyama, S. Tetrahedron 2010, 66, 9779.

[31] Möckel, R.; Babaoglu, E.; Hilt, G. Chem.-Eur. J. 2018, 24, 15781.

[32] (a) Broese, T.; Francke, R. Org. Lett. 2016, 18, 5896.

(b) Koleda, O.; Broese, T.; Noetzel, J.; Roemelt, M.; Suna, E.; Francke, R. J. Org. Chem. 2017, 82, 11669. 